\title{
El tipo de participación que promueve la escuela, una limitante para la inclusión
}

\section{The type of participation promoted in schools is a constraint factor for inclusive education}

\begin{abstract}
Azucena Ochoa Cervantes es docente investigadora de tiempo completo en la Universidad Autónoma de Querétaro (México) (azus@uaq.mx) (iD) (https://orcid.org/0000-0003-4515-9069)
\end{abstract}

Recibido: 2019-01-29 / Revisado: 2019-04-05 / Aceptado: 2019-05-20 / Publicado: 2019-07-01

\section{Resumen}

Diversos autores han mostrado que una de las barreras para el aprendizaje y la inclusión es la participación que se promueve dentro del contexto escolar,ya que a través de la participación se crean las condiciones para el aprecio a la diversidad y, por ende, para la inclusión. Sin embargo, en el contexto escolar la experiencia de participación a las que el alumnado tiene acceso son limitadas. Sobre esta base, se realizó un estudio con el objetivo de analizar el tipo de participación que se promueve en la escuela secundaria. Para alcanzar el objetivo, se aplicó un cuestionario a 539 estudiantes de nivel secundaria de escuelas públicas de la zona metropolitana de la ciudad de Querétaro, México. Los resultados muestran que si bien la escuela es el principal ámbito de participación que reconocen las y los estudiantes, sus ideas al respecto de la participación están acotadas a la emisión de una opinión que debe ser realizada de una manera específica, lo cual limita el aprendizaje y la inclusión.

Descriptores: Inclusión, educación inclusiva, participación, educación secundaria, barreras para la inclusión, niños, niñas y adolescentes.

\begin{abstract}
Several authors have shown that one of the barriers for learning and inclusion is the level of participation that is promoted within the school context, since through participation the conditions are created for the appreciation of diversity and, therefore, for the inclusion. However, in the school context, the participation experience to which students have access is limited. On this basis, a study was conducted with the objective of analyzing the type of participation promoted in secondary school. To achieve the objective, a questionnaire was applied to 539 middle school students from public schools in the metropolitan area of the city of Querétaro, Mexico. The results shown that although the school is the main area of participation that students recognize, their ideas regarding of their participation are limited to the issuance of an opinion that must be carried out in a specific way, which limits learning and inclusion.

Keywords: Inclusion, inclusive education, participation, secondary education, inclusion barriers, young children, teenagers.
\end{abstract}

Forma sugerida de citar: Ochoa Cervantes, A. (2019). El tipo de participación que promueve la escuela, una limitante para la inclusión. Alteridad, 14(2), 184-194. https://doi.org/10.17163/alt.v14n2.2019.03 


\section{Introducción y estado de la cuestión}

Los documentos normativos de la educación básica en México establecen como una prioridad la equidad educativa. Hablar de equidad nos remite necesariamente al clima escolar que se genera para crear ambientes propicios para el aprendizaje no sólo de conceptos, sino también de actitudes y valores que sustentan la sociedad democrática, esto implica que la escuela sea "un lugar de vida para el niño en el que éste sea un miembro de la sociedad, tenga conciencia de su pertenencia y a la que contribuya (Dewey, 2001, p. 224)

Partiendo de lo anterior, consideramos que la educación en la escuela debe proporcionar experiencias formativas que permitan una convivencia en la que todas y todos participen, ya que la participación asegura la inclusión.

La educación inclusiva hace referencia a un proceso educativo que busca que las niñas, niños y adolescentes puedan acceder a una educación de calidad, lo cual implicaría tomar en cuenta la diversidad de necesidades de las personas y esto se podría lograr a través del impulso la participación. Promover la educación inclusiva entonces debe considerar prioritariamente el aprecio por las diferencias lo cual requiere reconocer a cada una de las personas que integran los grupos escolares y a partir de esto, impulsar la participación para generar los aprendizajes. Esto necesariamente implica que las instituciones educativas privilegien prácticas organizativas y pedagógicas en las que las diferencias no se asuman como déficits y en donde la participación de la comunidad esté presente en todos los ámbitos de la vida escolar a través de diversos medios. permite:

La participación desde esta perspectiva

Aprender con otros y colaborar con ellos en el transcurso de las clases y las lecciones. Supone una implicación activa con lo que se está aprendiendo y enseñando y cabría decir lo mismo con relación a la educación que se está experimentado. Pero la participación también implica ser reconocido por lo que uno es y ser aceptado por esto mismo (Echeita, 2016, p. 4).

La importancia de la promoción de la participación infantil radica no sólo en que es un derecho jurídicamente establecido, sino que es un proceso que permitirá a la infancia desarrollar mejores aprendizajes y mejorar las condiciones de los ambientes en donde se desenvuelven. Aunado a lo anterior, la participación permite a las y los estudiantes el ejercicio de la ciudadanía activa pues es a partir de esta que se entra en relación con otros asumiendo responsabilidades para la mejora de la comunidad.

En un trabajo anterior (De la Concepción, 2015), describimos las tendencias que ha habido en el abordaje de las investigaciones o experiencias reportadas en torno a la participación infantil: la primera tiene que ver con la participación desde el reconocimiento de los derechos (IFE, 2007; Zanabria, Fragoso, \& Martínez, 2007); la segunda se relaciona con los trabajos que muestran que con la participación infantil, la infancia desarrolla la capacidad de influir y transformar el entorno en el que viven (Muñoz, 2003; UNICEF s.f; Valderrama, 2013). La tercera tendencia en la investigación es la que considera la participación como componente nuclear entre los conceptos de ciudadanía y democracia (Acosta, \& Pineda, 2007; Botero, Torres, \& Alvarado, 2008; Cajiao, 1998; Van Dijk, 2007; Hart, 1993; Fernández, 2009; Lansdown, 2001; Trilla, \& Novella, 2011). Por último, se identifican los estudios que tiene que ver con los significados o concepciones que los protagonistas le confieren al término participación (Carmona, 2008; Bosch, \& GonzálezMontfort, 2012; Hernández, 2014; Ochoa Salinas, \& Díez-Martínez, 2014; Ochoa, 2015). Dentro de esta última aproximación se insertan los resultados del presente estudio.

A partir de la revisión anterior, afirmamos que para que la infancia pueda ejercer su participación es prioritario que se creen las condiciones para que se lleve a cabo, específicamente en el con- 
texto escolar pues además de su proximidad, es el contexto reconocido por las niñas, niños y adolescentes (NNA) como el principal ámbito de participación; por lo que es necesario que la escuela enseñe a participar desarrollando habilidades, conocimientos y actitudes que permitan el diálogo y la asociación. Sin embargo, para lograr esto se necesita la creación de un clima de confianza en donde se establezcan relaciones horizontales entre los adultos encargados y las NNA. Lo anterior implicaría desterrar creencias acerca de la participación pues en estudios anteriores se ha podido constatar que las actividades pedagógicas en las escuelas son rígidas y las formas de participación tienen que ver con la emisión de una respuesta o con el seguimiento de las instrucciones dadas por los docentes (Cerda, Loreto, Magendzo, Cruz, \& Varas, 2004; De la Concepción, 2015).

Partiendo de la idea de que en la escuela se promueve la participación es necesario reflexionar acerca de si las formas de participación garantizan la inclusión educativa pues se caracterizan por una alta direccionalidad y rigidez.

Tipologías para observar - e impulsar- la participación de NNA

Algunos autores y organismos (Hart, 1993; Trilla, \& Novella, 2001; Gaitán, 1998, OEA/ INN, 2010) han propuesto algunas formas $y$ parámetros útiles a través de los cuales sería posible observar la participación de los niños, niñas y adolescentes; estas propuestas pueden servir como indicadores generales para promover la participación escolar en un clima de respeto y aprecio por la diversidad.

El menú de indicadores y sistema de monitoreo del derecho a la participación de niños, niñas $y$ adolescentes considera que la participación infantil es un proceso que para sea efectivo debería considerar tres momentos: la información, la construcción y reconocimiento de la opinión y la expresión y escucha. Por lo que se requiere proporcionar información accesible y pertinente, permitir y respetar las opiniones y mantener la escucha activa para que se puedan expresar esas opiniones (OEA/INN, 2010).

Roger Hart (1993) hace una propuesta de análisis con el objetivo de reflexionar sobre los alcances de la participación de los niños en los proyectos comunitarios. Utiliza la metáfora de una escalera para describir gradualmente la participación infantil. El primer peldaño en la escalera de Hart hace referencia a la manipulación; el segundo peldaño es el de la decoración, en que, por ejemplo, los niños y niñas "usan camisetas" relacionadas con alguna causa, aunque no sepan de qué se trata y por ende no participan, sólo "están". Como tercer peldaño se encuentra la participación simbólica, ésta se caracteriza porque puede ser que se permita que las NNA se expresen, pero esta expresión no influye en el proyecto. A los tres peldaños anteriores, el autor los denomina como "no participación".

Continuando de manera ascendente en la escalera encontramos el cuarto peldaño, el de asignado pero informado, en el cual las y los participantes saben los objetivos del proyecto, saben sobre su papel en el mismo y que tienen la oportunidad de involucrarse de manera voluntaria después de tener la información suficiente para tomar esta decisión. En el quinto peldaño, consultados e informados, los niños y niñas se convierten en consultores de los responsables del proyecto. El sexto peldaño denominado proyectos iniciados por los adultos se trata de que las decisiones sobre las actividades se compartan con los niños y niñas. El séptimo peldaño es el denominado proyectos iniciados y dirigidos por los niños, y finalmente, el nivel más alto de participación en la escalera, el octavo peldaño de los proyectos iniciados por los niños en que las decisiones se comparten con los adultos (Hart, 1993).

Basados en la tipología anterior, Trilla y Novella (2001) proponen cuatro clases de participación de la infancia:

Participación simple, consistente en tomar parte en una actividad como espectador sin que la infancia intervenga en la preparación ni en el contenido o desarrollo. Se limitan a seguir indicaciones. 
Participación consultiva, implica tomar opinión del alumnado en asuntos de su competencia o interés, esto supone una escucha activa por parte de los adultos.

Participación proyectiva, la infancia se convierte se hace responsable del proyecto y se convierte en actor del cambio; este tipo de participación es más compleja y puede presentarse en diferentes momentos del proyecto.

Metaparticipación, consiste en que el alumnado reivindique su derecho a participar, lo cual requiere la creación de mecanismos y espacios de participación, así como un clima de confianza que lo permita.

Los autores hacen énfasis que los niveles no son progresivos, sino que pueden presentarse avances y retrocesos. A su vez, Gaitán (1998) concibe la participación infantil como un proceso tendiente a incrementar el poder de la niñez organizada en su relación con los adultos y propone tres indicadores, con sub-niveles, para observarla:

Conciencia, se refiere a la capacidad de las niñas, niños y adolescentes de entender su derecho a participar "Cuanta más conciencia tengan mayor será su participación” (p. 91). A su vez este indicador se compone por los subniveles: ninguna conciencia, conciencia intuitiva, conciencia básica y conciencia profunda.

Capacidad de decisión, es poder valorar las alternativas que existen dentro del proyecto, los subniveles que componen este indicador son: ninguna opción, opción única, opción relativa, opciones múltiples.

Capacidad de acción, este observable se refiere a las actividades que fueron impulsados por los participantes, este indicador presenta lo subniveles: acción impuesta, acción conducida, acción negociada y acción de vanguardia.

A diferencia de los autores anteriores, para Gaitán (1998) estos indicadores se relacionan entre sí y el orden también es importante, ya que determina la dirección del camino a recorrer. Un mayor nivel de conciencia se traduce en más capacidad de decisión y una mejor calidad de acción.
Estas tipologías podrían ser útiles no sólo para observar sino para generar las condiciones para que se propicie una participación auténtica y al mismo tiempo para promover la inclusión ya que la falta de participación es una barrera para la misma.

Para que se promueva la participación es importante propiciar un clima de confianza que permita la expresión y el intercambio de ideas, pensamientos y sentimientos, así mismo que se pongan en marcha estrategias metodológicas que se sustenten en la pedagogía activa y en el aprendizaje basado en la experiencia, lo que implicaría la construcción colectiva del conocimiento.

Con base en lo planteado, en este trabajo tenemos como objetivo analizar las condiciones para la participación que se dan —o no- en las escuelas secundarias a partir de lo que dicen los estudiantes.

\section{Material y métodos}

Se realizó un estudio cualitativo siguiendo el método de casos. Este método permite la descripción a profundidad de las múltiples causas que generan la problemática planteada, pues "las acciones humanas importantes pocas veces tienen una causa simple" (Stake, 2010, p. 43). En este método se emplean varias técnicas de recolección de datos que permiten recopilar las voces y escenarios implicados en el caso que se estudia. Al respecto, Hernández, Fernández y Baptista (2006) lo definen como "estudios que al utilizar los procesos de investigación cuantitativa, cualitativa o mixta analizan profundamente una unidad para responder al planteamiento del problema” (p. 224).

De acuerdo a su objetivo, el presente estudio de casos fue descriptivo, pues lo que se busca es caracterizar las condiciones en que se da la participación en escuelas secundarias.

La muestra estuvo constituida por 539 estudiantes de secundaria de dos escuelas públicas de la ciudad de Querétaro, México, de los cuales 183 eran de primer grado, 178 de segundo y 178 de tercero. 
El instrumento fue un cuestionario para indagar las condiciones en las que se da la participación del alumnado a partir de las experiencias participativas que dicen vivir en su escuela, el instrumentos se estructuraba en los siguientes apartados: el primero con datos generales, el segundo describía cuatro situaciones con problemáticas que les afectaban cotidianamente, en este apartado escribían, además de su opinión, lo que ellos harían en esa situación; el tercer apartado estuvo compuesto por quince preguntas abiertas que indagaban sobre los siguientes aspectos:

Concepciones de las niñas y niños sobre participación: Cuando escuchas la palabra participar, ¿en qué piensas?, ¿en qué lugares puedes participar?, ¿cómo participas?

Concepciones de las niñas y niños sobre la participación en el contexto escolar: ¿En tu escuela puedes participar?, ¿ cómo participas en tu escuela?

Concepciones de las niñas y niños sobre la participación infantil como derecho civil y político: ¿Participar es un derecho?, ¿por qué participar es un derecho de las niñas y niños?

Las respuestas fueron transcritas y los datos del cuestionario se tabularon en categorías para posteriormente hacer un análisis global, es decir, una comparación de los datos con los referentes teóricos.

\section{Análisis y resultados}

Como se mencionó en la descripción de la metodología, el cuestionario fue aplicado a las y los estudiantes, y dado que las preguntas eran abiertas, éstas se transcribieron y se organizaron a partir de su semejanza conceptual. Como una respuesta podía contener varias ideas, se procedió a ubicarlas en categorías, posteriormente se contabilizaron y se calcularon porcentajes para observar las tendencias en las respuestas, por lo que el porcentaje que se presenta en las tablas no corresponde al número de participantes sino a los tipos de respuesta.

La primera pregunta tenía que ver con indagar las ideas que tiene el alumnado acerca de la participación, se les planteó la pregunta: Cuando escuchas la palabra participar, ¿en qué piensas? Como se puede observar en la Tabla 1, la idea de participación que presentan tiene que ver con la emisión de una opinión, seguido de hablar o decir. Llama la atención también que la respuesta que tiene la siguiente proporción es la de no sé y otras. Esto es importante pues nos puede indicar que esta población no tiene clara una de las condiciones necesarias para que se promueva la participación, el reconocimiento del derecho a hacerlo.

Tabla 1. Porcentajes de tipos de respuesta a la pregunta Cuando escuchas la palabra participar, ¿en qué piensas?

\begin{tabular}{|l|c|}
\hline \multicolumn{1}{|c|}{ Categorías } & $\%$ \\
\hline Dar una opinión & 40 \\
\hline Hablar o decir & 16 \\
\hline Otras & 10 \\
\hline No sé/ No contestó & 10 \\
\hline Participar & 9 \\
\hline Es un derecho & 6 \\
\hline Levantar la mano & 4 \\
\hline Ayudar & 4 \\
\hline Actividades escolares & 1 \\
\hline
\end{tabular}

Fuente: Elaboración propia. 
Con el fin de profundizar acerca del reconocimiento a la participación como un derecho, la siguiente pregunta era si consideraban a la participación como un derecho y el por qué lo consideraban así. El $100 \%$ de los participantes consideró que la participación es un derecho, este dato es relevante pues para que un derecho se haga efectivo es necesario su reconocimiento.

En relación con las razones por las que consideraban que participar es un derecho en su mayoría se concentraban en porque les permite expresar una opinión (79\%); si relacionamos este resultado con el inmediato anterior podemos observar que la concepción de participación está circunscrita a la expresión o emisión de una opinión. Sin embargo, aparecen algunas respuestas que aluden a otros aspectos, como es el caso de porque somos iguales (13\%), el $8 \%$ restante aludía a que es una obligación.

Posteriormente se indagó acerca de los ámbitos de participación que distinguen los estudiantes.

Tabla 2. Porcentajes de tipos de respuesta a la pregunta ¿En qué lugares consideras que puedes participar?

\begin{tabular}{|l|c|}
\hline \multicolumn{1}{|c|}{ Categorías } & $\%$ \\
\hline Escuela & 30 \\
\hline Casa & 28 \\
\hline En todos lados & 24 \\
\hline Otros & 7 \\
\hline Aula & 5 \\
\hline No sé/ No contestó & 5 \\
\hline En el lugar apropiado & 1 \\
\hline
\end{tabular}

Fuente: Elaboración propia.

Las respuestas de la Tabla 2 indican que la escuela se distingue como el ámbito privilegiado de participación, seguido de la casa. Lo anterior resulta de fundamental importancia pues la escuela, dada la proximidad a los estudiantes, podría promover experiencias participativas que implicaran la construcción de ambientes inclusivos de aprendizaje y convivencia.

Con la intención de indagar acerca de la iniciativa que podrían tener o no los estudiantes para ejercer la participación, se cuestionó acerca de cuándo participaban. La mayor parte de las respuestas se concentró en la categoría siempre con un $28 \%$, seguida de cuando me interesa o quiero con un $25 \%$, cuando me lo piden $20 \%$, cuando es necesario $12 \%$ y actividades escolares $12 \%$. Llama la atención estas respuestas pues denotan claramente el factor motivación como importante para favorecer la participación; lo anterior habría que tenerlo en cuenta para promover metodologías activas.

Para indagar acerca de las experiencias en participación que decían tener los estudiantes, se les preguntó la forma en que participan. Las respuestas en su mayoría referían a dando mi opinión 57\%, ayudando 11\%, hablando $10 \%$, levantando la mano $8 \%$, otras $8 \%$ y cuidando las formas con un $6 \%$. Se observa que la forma en que los estudiantes distinguen que participan es dando su opinión seguida de ayudando y hablando. Relacionando estas respuestas con las presentadas en la Tabla 1 se puede confirmar que la idea de participación que presentan los participantes es simple (Trilla, \& Novella, 2001) o una participación simbólica (Hart, 1993).

Para indagar específicamente acerca del contexto escolar, se preguntó acerca de cómo se participa en la escuela. Las respuestas se presentan en la Tabla 3. 
Tabla 3. Porcentajes de tipos de respuesta a la pregunta ¿Cómo participas en la escuela?

\begin{tabular}{|l|c|}
\hline \multicolumn{1}{|c|}{ Categorías } & $\%$ \\
\hline Dando mi opinión & 38 \\
\hline Levantando la mano & 23 \\
\hline Cuidando las formas & 14 \\
\hline Ayudando & 10 \\
\hline Hablando & 9 \\
\hline Actividades escolares & 6 \\
\hline
\end{tabular}

Fuente: Elaboración propia.

A diferencia de las respuestas de la pregunta anterior, la respuesta levantando la mano aumenta y disminuye la respuesta dando mi opinión. Llama la atención la emergencia de la categoría que denominamos como cuidando las formas, dado que aquí se incluyeron respuestas acerca de cómo se espera que sea la participación en la escuela por ejemplo con voz fuerte y clara, sin nervios, con seguridad, entre otras. La emergencia de este tipo de respuestas denota la influencia de las creencias de los adultos acerca de la participación y su implicación en la puesta en marcha de actividades y/o estrategias en donde se impulsen diversas formas de participación.

Para sondear específicamente acerca de cómo se da la participación en el aula se preguntó acerca de este espacio en particular. Las respuestas se presentan en la siguiente tabla.

Tabla 4. Porcentajes de tipos de respuesta a la pregunta ¿Cómo participas en tu salón?

\begin{tabular}{|l|c|}
\hline \multicolumn{1}{|c|}{ Categorías } & $\%$ \\
\hline Dando mi opinión & 37 \\
\hline Levantando la mano & 26 \\
\hline Actividades escolares & 21 \\
\hline Doy propuestas o ideas & 10 \\
\hline Hablando & 3 \\
\hline Ayudando & 3 \\
\hline
\end{tabular}

Fuente: Elaboración propia.

En este caso, el mayor porcentaje de respuestas se concentra en dando mi opinión, seguido de levantando la mano y actividades escolares. Este tipo de respuesta incluía actividades que tenían que ver con exposiciones, pasar al pizarrón, leer, contestar preguntas puntuales, es decir, ejecutar indicaciones del docente para completar alguna tarea escolar.

Para tratar de advertir acciones específicas de participación, se les cuestionó acerca de las actividades en las que han sido invitados en la escuela. El mayor porcentaje de respuestas se concentra en ninguna (35\%). La siguiente tendencia en las respuestas se refiere a las actividades escolares $(21 \%)$, por lo que se deduce que la población de este estudio identifica a la participación con la ejecución de acciones propiamente escolares. Las siguientes tendencias en las respuestas aludían a actividades deportivas (16\%), culturales (13\%), no contestó (9\%) y otras con un $6 \%$.

Como se dijo, para poder promover una participación auténtica es necesario involucrar a las y los estudiantes en las problemáticas que les 
afectan, para que en su momento se involucren también en su resolución. Para conocer cuáles son las problemáticas que observan, se les pidió que describieran una situación que les preocupara en su escuela. Las respuestas se presentan enseguida.

Tabla 5. Porcentajes de tipos de respuesta a la pregunta Escribe una situación que te preocupe de tu escuela

\begin{tabular}{|l|c|}
\hline \multicolumn{1}{|c|}{ Categorías } & $\%$ \\
\hline Trato entre alumnos & 21 \\
\hline Actitud de los docentes & 20 \\
\hline Instalaciones escolares & 17 \\
\hline Situaciones de riesgo (inseguridad, drogadicción, delincuencia) & 12 \\
\hline Limpieza & 9 \\
\hline Sexualidad, género & 9 \\
\hline No sé/No contestó & 8 \\
\hline No hay ninguno & 2 \\
\hline Calificaciones/Reprobar & 2 \\
\hline
\end{tabular}

Fuente: Elaboración propia.

Lo que más preocupa a los estudiantes es el trato entre alumnos, seguido de la actitud de los docentes. Se puede inferir, a partir de estas respuestas, que posiblemente existen conflictos de convivencia en estos grupos, ya que las dos problemáticas con mayores porcentajes de respuestas aluden a ello.
Para profundizar en lo anterior, se les cuestionó acerca de la resolución de esa problemática y su participación como actores en la solución. En la siguiente tabla se ofrecen las respuestas.

Tabla 6. Porcentaje de tipos de respuesta a la pregunta ¿Cómo la resolverías?

\begin{tabular}{|l|l|}
\hline \multicolumn{1}{|c|}{ Categorías } & \multicolumn{1}{c|}{$\%$} \\
\hline Sancionando & 17 \\
\hline Dando aviso a una autoridad & 15 \\
\hline Hablando/opinando & 15 \\
\hline Participando & 12 \\
\hline Medidas de Seguridad & 11 \\
\hline Ayudando & 9 \\
\hline Otras & 8 \\
\hline Limpiando & 8 \\
\hline Arreglando/Haciendo & 5 \\
\hline & \\
\hline
\end{tabular}

Fuente: Elaboración propia.

Como se observa en la Tabla 6, la manera en que se concibe resolver la problemática es prioritariamente sancionando, seguida de dando aviso a la autoridad. Estas respuestas resultan reveladoras y confirman lo mostrado a lo largo de las tablas anteriores, las y los estudiantes de estos grupos tienen una concepción acotada de la participación y una actitud pasiva dado que 
en las escuelas las experiencias de participación son limitadas a actividades escolares dirigidas por el o la docente, esto trae como consecuencia que el alumnado asuma un rol de espectador que piensa en opciones heterónomas para resolver los conflictos que le preocupan.

\section{Discusión y conclusiones}

Los documentos normativos y los discursos políticos reconocen la importancia de impulsar una educación inclusiva, sin embargo, estas intenciones pocas veces se ven traducidas en prácticas congruentes dentro de las instituciones.

A partir de los resultados se puede observar que las ideas de participación que menciona el alumnado se relacionan con la emisión de una opinión, también podemos afirmar que, si bien existe participación en la escuela, es una participación acotada y limitada a actividades escolares, pensadas y planeadas por los adultos, lo que se llama una participación simple (Trilla, \& Novella, 2001). Coincidimos con Gaitán (1998) en relación con que es necesario impulsar actividades participativas que permitan a las niñas, niños y adolescentes un mayor nivel de conciencia acerca de sus decisiones y de sus acciones. Sin embargo, esto implicaría que las y los estudiantes en la escuela dejaran de ser espectadores y meros ejecutores de órdenes.

En este sentido, y como se observó en los resultados, si las y los estudiantes consideran que la participación se limita a la emisión de una opinión y que ésta debe ser bajo los cánones establecidos por la institución, están asumiendo un rol pasivo, lo que no favorece la promoción de una participación auténtica. Por tanto, es necesario generar un clima que promueva actividades diversas en que no se privilegie una sola forma de participación - la oral-, como regularmente sucede en nuestras escuelas, lo cual genera mecanismos de exclusión pues son regularmente los mismos quienes toman la palabra, cuando se la otorgan. Al respecto, Corona y Morfín (2001) afirman que:
La expresión verbal es solamente una y tal vez no la más importante de las múltiples formas que tienen los niños para manifestar lo que está pasando en su interior. Los actos, las expresiones emocionales incluyendo los berrinches, las actitudes y el silencio están llenos de sentido y se requiere agudizar la sensibilidad para entender su significado. (p. 44)

A partir de estos resultados constatamos que la experiencia que se vive en la escuela en relación con la participación se acota al ámbito académico y es dirigida por las y los docentes, pues las actividades cotidianas son estructuradas y organizadas por los adultos, asignando un rol pasivo al alumnado. Esto se confirma contrastando trabajos anteriores (Ochoa, 2015; Ochoa, Pérez, \& Salinas, 2018). Al respecto, Bolívar (2016) menciona que:

La estructura institucional $\mathrm{u}$ organizativa de un centro escolar proporciona un conjunto de tareas, procesos y significados en la vida diaria que están promoviendo una determinada ciudadanía. El aprendizaje de valores y actitudes exige, entonces, experimentarlos en la trama organizativa de la escuela o establecimiento escolar, por lo que hay formas de organización que institucionalizan mejor educar para una sociedad democrática. Una escuela para aprender a ejercer la ciudadanía ha de promover, en primer lugar, la participación activa en todos los niveles. (p. 79)

Una de las barreras para la inclusión es el tipo de participación que se promueve y el no reconocimiento de que la participación de las niñas, niños y adolescentes es un derecho implica que no se creen las condiciones para su ejercicio. Más aún: si los propios niños, niñas y adolescentes no lo reconocen como tal les será difícil exigir su cumplimiento. De ahí la importancia de que se resguarde y se promueva.

Es así que, para favorecer la participación de los niños, niñas y adolescentes, y con ello generar ambientes de respeto y aprecio por la diversidad, se requiere un cambio de mirada de los adultos acerca de la infancia y acerca de su 
participación dentro de la escuela que considere tres aspectos:

- Lo pedagógico, implementando metodologías participativas que impacten en el aprendizaje.

- Lo cultural, pues implica dar voz a los niños y niñas y con esto redistribuir el poder de la toma de decisiones.

- La promoción de los derechos. La participación no debe ser considerada como una concesión de los adultos sino como un derecho que hay que respetar.

De acuerdo al Modelo de la Educación Básica Obligatoria (SEP, 2017) propuesto por el Estado mexicano, así como de diversos organismos internacionales como la UNESCO (2015), la inclusión y la equidad son "la piedra angular de una educación transformadora” (p. 7), lo que implicaría la puesta en marcha de estrategias participativas, así como crear las condiciones y mecanismos necesarios para que todos y todas participen.

\section{Agradecimientos}

Se agradece el financiamiento parcial para esta investigación al Fondo para el Fortalecimiento de la Investigación de la Universidad Autónoma de Querétaro.

\section{Referencias bibliográficas}

Acosta, A., \& Pineda, N. (2007). Ciudad y participación infantil. Bogotá: CINDE.

Bolívar, A. (2016). Educar democráticamente para una ciudadanía activa. Revista Internacional de Educación para la Justicia Social, 5(1), 69-87.

Bosch, D., \& González-Montfort, N. (2012). ¿Cómo perciben los alumnos su participación en los centros de secundaria? Una investigación sobre las representaciones sociales de los alumnos. En De Alba, N., García, F. y Santisteban, A. (Eds.), Educar para la participación ciudadana en la enseñanza de la Ciencias Sociales, vol. I. Sevilla: Diada/
Asociación Universitaria de Profesorado de Didáctica de las Ciencias Sociales.

Botero, P., Torres, J., \& Alvarado, S. (2008). Perspectivas teóricas para comprender la categoría participación ciudadana-política juvenil en Colombia. Revista Latinoamericana de Ciencias Sociales, Niñez y Juventud, 6(2). Manizales: CINDE.

Cajiao, F. (1998). Niños y jóvenes como ciudadanos de pleno derecho. En La participación de niños y adolescentes en el contexto de la convención: visiones y perspectivas (pp. 17-32). Bogotá: Actas del Seminario UNICEF.

Carmona, D. (2008). Concepciones de ciudadanía en niños y niñas del municipio de Marquetalia Caldas. Tesis de maestría. Manizales: Centro de Estudios Avanzados en Niñez y Juventud/ Universidad de Manizales/CINDE.

Cerda, A., Loreto, M., Magendzo, A., Cruz, E., \& Varas, R. (2004). El complejo camino de la formación ciudadana: una mirada a las prácticas docentes. Santiago de Chile: LOM/PIIE.

Corona, Y., \& Morfín, M. (2001). Diálogos de saberes sobreparticipación infantil. Ciudad de México: Universidad Autónoma Metropolitana, Unidad Xochimilco.

Dewey, J. (2001). Democracia yeducación. Introducción a la filosofía de la educación. Madrid: Morata.

Echeita, G. (2016). Educación Inclusiva. De los sueños a la práctica del aula. Cuadernos de Educación, 75. Universidad Alberto Hurtado. (https://bit.ly/2Q2tooX).

Fernández. J. (2009). Los niños y niñas: ¿Ciudadanos de hoy o de mañana? Alternativas. Cuadernos de Trabajo Social, 16, 11-126.

Gaitán, A. (1998). Protagonismo infantil. En B. Ablegglen y R. Benes (Comps.), La participación de los niños y adolescentes en el contexto de la convención sobre los derechos del niño: visiones y perspectivas. Bogotá: UNICEF.

Hart, R. (1993). La participación de los niños, de la participación simbólica a la participación auténtica. Ensayos Innocenti, 4. UNICEF.

Hernández, M. A. (2014). Des-encuentros de la participación infantil en el ámbito escolar. Un acercamiento a las ideas de los docentes de educación primaria de escuelas públicas de la $\mathrm{Cd}$. de Querétaro. Tesis de Maestría en Educación 
para la Ciudadanía. México: Universidad Autónoma de Querétaro.

Hernández, R., Fernández, C., \& Baptista, P. (2006). Metodología de la investigación. Enfoques cuantitativo, cualitativo y mixto. México: McGraw Hill.

IFE (2007). Experiencias nacionales de participación infantil y juvenil 1997-2006. México: IFE.

Lansdown, G. (2001). Promoting Children's Participation in Democratic DecisionsMaking. Siena: UNICEF.

Muñoz, D. (2003). (coord.). Participar también es cosa de niños. Guía didáctica para el profesorado. Comunidad Valenciana: UNICEF.

Ochoa, A. (2015). Concepciones sobre participación de niñas, niños y adolescentes: su importancia en la construcción de la convivencia escolar. Cultura, Educación y Sociedad, 6(2), 9-28.

Ochoa, A., Pérez, L., \& Salinas, J. (2018). El aprendizaje servicio como estrategia expansiva y transformadora. Revista Iberoamericana de Educación, 76, 15-34.

Ochoa, A., Salinas, J. y Díez-Martínez, E. (2014). La participación de niñas, niños y adolescentes, condición indispensable para la construcción de la convivencia en escuelas. En A. Calvo, C. Rodríguez-Hoyos, e I. Rodríguez (Coords.), Investigar para acompañar el cambio educativo y social. El papel de la universidad. Santander: AUPOF/Universidad de Cantabria.

OEA/INN (2010). Menú de indicadores y sistema de monitoreo del derecho a la participación de niños, niñas y adolescentes. Montevideo, Uruguay: OEA-IIN. (https://bit.ly/2Wakwzk).

SEP (2017). Modelo educativo para la educación obligatoria. Educar para la libertad y la creatividad. México: Secretaría de Educación Pública.

Stake, R. (2010). Investigación con estudio de casos. Madrid: Ediciones Morata.

Trilla, J., \& Novella, A. (2001). Educación y participación social de la infancia. Revista Iberoamericana de Educación, 26. (https:// bit.ly/2Tl1X9O).

Trilla, J. \& Novella, A. (2011). Participación, democracia y formación para la ciudadanía. Los consejos de la infancia. Revista de educación, $356,23-43$.

UNICEF (s. f). Manual para el profesorado. La voz de la infancia. (https://bit.ly/2WaWnZr).

UNESCO (2015). Declaración de Incheon y Marco de Acción para la realización del Objetivo de Desarrollo Sostenible 4. Garantizar una educación inclusiva, equitativa y de calidad y promover actividades de aprendizaje permanente para todos. (https://bit.ly/2AQmz1Z).

Valderrama, R. (2013). Los procesos de participación como un espacio educativo de desarrollo de la pedagogía. Cuestiones pedagógicas, 22, 351-376.

Van Dijk, S. (2007). Participación infantil. Una revisión desde la ciudadanía. Tramas, 28, 43-66.

Zanabria, M., Fragoso, B., \& Martínez, A. (2007). Experiencias de participación infantil en Tlaxcala y Ciudad de México. Tramas, 28, 121-140. 